\title{
openheart Early metabolic response to acute myocardial ischaemia in patients undergoing elective coronary angioplasty
}

\author{
Sara Di Marino, ${ }^{1}$ Nicola Viceconte,${ }^{2}$ Angelo Lembo, ${ }^{1}$ Vincenzo Summa, ${ }^{1}$ \\ Gaetano Tanzilli, ${ }^{2}$ Valeria Raparelli, ${ }^{3}$ Giovanni Truscelli, ${ }^{2}$ Enrico Mangieri, ${ }^{2}$ \\ Carlo Gaudio, ${ }^{2}$ Daniel Oscar Cicero ${ }^{1,4}$
}

To cite: Di Marino S, Viceconte N, Lembo A, et al. Early metabolic response to acute myocardial ischaemia in patients undergoing elective coronary angioplasty. Open Heart 2018;5: 000709 . doi:10.1136/ openhrt-2017-000709

SDM and NV contributed equally.

Received 30 August 2017 Revised 9 February 2018 Accepted 14 February 2018

A) Check for updates

${ }^{1}$ IRBM Science Park S.p.A., Pomezia, Italy

${ }^{2}$ Department of Cardiovascular, Respiratory, Nephrologic, Anaesthesiologic and Geriatric Sciences, Sapienza University of Rome, Policlinico Umberto I, Rome, Italy

${ }^{3}$ Department of Experimental Medicine, Sapienza University of Rome, Policlinico Umberto I Rome, Italy

${ }^{4}$ Department of Chemical Science and Technology, Università di Roma "Tor Vergata", Rome, Italy

Correspondence to Prof. Daniel Oscar Cicero; cicero@scienze.uniroma2.it

\section{ABSTRACT}

Objective Balloon-induced transient coronary ischaemia represents a model of myocardial ischaemia and reperfusion. We are interested in the very early systemic metabolic response to this event.

Methods Blood samples of patients with stable angina (SA) were collected before and after coronary angioplasty. Serum metabolic profiles were obtained using nuclear magnetic resonance spectroscopy. Univariate and multivariate analyses were used to investigate changes in metabolite concentrations.

Results Thirty-four consecutive patients with SA, undergoing elective coronary angioplasty at Policlinico Umberto I of Rome, were included in this study. Changes in metabolites concentration induced by balloon occlusion in venous and arterial sera were detected. In both serum types, a significant increase in ketone bodies, 2-hydroxybutyrate, glutamine and 0 -acetylcarnitine concentration is observed, while alanine, lactate, phenylalanine and tyrosine decreased after intervention. Most significant metabolic changes were detected in arterial serum.

Conclusions Our study points out two main global metabolic changes in peripheral blood after ballooninduced coronary ischaemia: ketone bodies increase and lactate decrease. Both could be related to compensation mechanisms finalised to fulfil heart's needs after short period of myocardial ischaemia and probably after reperfusion.

\section{INTRODUCTION}

Despite improvements in percutaneous coronary intervention (PCI) and the development of advanced pharmacological approaches, ischaemic heart disease persists as a major worldwide health issue. Indeed ischaemic heart disease is the leading cause of morbidity and mortality for both men and women. ${ }^{1}$ A better understanding of the pathophysiological mechanisms behind ischaemia may improve opportunities for the early identification of the disease and thus allow implementation of cardiovascular preventive strategies. While prolonged myocardial ischaemia may be biochemically profiled by its associated release of cell injury

\section{Key questions}

What is already known about this subject?

- Although there are a significant number of papers describing the changes that occur locally in the heart during ischemia, data on whole body response to this challenging condition are much rare. Data on circulating lactate and other metabolites were investigated after balloon occlusion, but results were partially contradictory.

What does this study add?

- This study presents a metabolomics approach to measure the levels of key metabolites that change in response to the ischemia induced by balloon occlusion. The observed effects are rationalized in terms of a compensation mechanism of the whole body to fulfil the needs of the heart.

How might this impact on clinical practice?

- Identification of a specific metabolic change induced by myocardial ischemia could be of clinical relevance to rule out coronary events in patients referring for acute chest pain in emergency departments.

markers, early molecular and cellular events resulting from short periods of ischaemia remain largely uncharacterised. Several potential biomarkers for early myocardial ischaemia were recently reported but so far none has sufficient diagnostic accuracy to justify their use in a clinical setting. ${ }^{2}$

Metabolomics allows a quantitative measurement of human metabolism by analysing simultaneously dynamic changes in many metabolites. Knowledge of the metabolic profile changes associated with early myocardial ischaemia could therefore have a huge impact on decision making in response to cardiac events in an emergency setting. Additionally, the ability to identify subclinical ischaemia could have important implications 
in daily practice with respect to resource optimisation and the avoidance of inappropriate and potentially dangerous discharges of patients from care.

Balloon-induced transient ischaemia represents a safe and reproducible model for myocardial ischaemia and reperfusion. During ischaemia, energy production is impaired due to lack of oxygen supply and activation of mechanisms for oxygen-independent ATP production is the first cell response, a process that is further complicated by myocardial reperfusion, which potentially increases cell injury.

The aim of this investigation is to understand the very early systemic metabolic response to myocardial ischaemia, through a nuclear magnetic resonance (NMR) spectroscopy analysis performed on peripheral blood samples collected before and after balloon-mediated transient ischaemia.

\section{METHODS}

The present study included patients from both sexes aged $\geq 18$ years that were undergoing coronary angiography and elective coronary angioplasty for stable angina (SA) at the Policlinico Umberto I of Rome between September 2015 and May 2016. Patients had stress test-inducible myocardial ischaemia and de novo native coronary lesions. Clinical and pharmacological information was recorded for each patient at the time of his PCI. Blood samples were collected in accordance with the ethical guidelines, and patients provided written consent. A $10 \mathrm{~mL}$ sample of arterial and venous blood was collected from each patient immediately before and $10 \mathrm{~min}$ after $60 \mathrm{~s}$ intracoronary balloon inflation. After collection, the whole blood coagulated. The clothing was removed by centrifugation at $3000 \times \mathrm{g}$ for $10 \mathrm{~min}$ in a refrigerated centrifuge. The resulting supernatant (serum) was stored at $-80^{\circ} \mathrm{C}$.

\section{Sample preparation}

Serum samples were thawed in ice and deproteinised by centrifuging with $3 \mathrm{kDa}$ cut-off Amicon Ultra centrifuge filter devices ( $3500 \mathrm{~g}$ for 1 hour at $4^{\circ} \mathrm{C}$ ). Filter membranes were prewashed with distilled $\mathrm{H}_{2} \mathrm{O}$ to remove glycerol. After centrifugation, filtrates were checked visually for red tint, which indicates that the membrane was compromised, and in those cases the filtration procedure was repeated. To each $400 \mu \mathrm{L}$ of filtered serum, $100 \mu \mathrm{L}$ of NMR buffer ( $250 \mathrm{mM}$ phosphate buffer, $\mathrm{pH}$ 7.4, containing $1.1 \mathrm{mM}$ trimethylsilyl propanoic acid-d4 (TSP), $10 \% \mathrm{D}_{2} \mathrm{O}, 2 \% \mathrm{NaN}_{3}$ ) were added. Each serum sample was mixed with vortex, and $50 \mu \mathrm{L}$ of solution was transferred to a $1.7 \mathrm{~mm}$ NMR microtube.

\section{NMR spectroscopy}

All ${ }^{1} \mathrm{H}-\mathrm{NMR}$ spectra were acquired using a Bruker Avance $600 \mathrm{MHz}$ equipped with a $1.7 \mathrm{~mm}$ inverse probe, Z-gradients and Sample-Jet autosampler. For each serum sample, a ${ }^{1} \mathrm{H}-\mathrm{NMR}$ spectrum was obtained with water peak suppression using a standard pulse sequence (noesypr1d), 2048 scans, four dummy scans, a spectral width of $12 \mathrm{ppm}$, an acquisition time of $2 \mathrm{~s}$, a relaxation delay of $3 \mathrm{~s}$ and a mixing time of $100 \mathrm{~ms}$. All spectra were acquired at $25^{\circ} \mathrm{C}$.

\section{Metabolite identification and quantification}

Spectra were processed and analysed using the Chenomx NMR Suite Professional software V.8.1. All spectra were zero-filled to $128 \mathrm{~K}$ data points and subjected to line broadening of $0.5 \mathrm{~Hz}$. TSP was set as internal standard at $0.22 \mathrm{mM}$ concentration. Chenomx software allowed the identification and quantification of 41 metabolites in each spectrum. Metabolite concentrations were normalised according to the method of probabilistic quotient. ${ }^{4}$

\section{Statistical analysis}

As the present research was designed as a pilot study, a number of subjects between 24 and 50 was deemed suitable based on previous literature precedent. ${ }^{5}$

Variables with normal distribution were expressed as mean and SD, and tested for significant differences with the Student's t-test. Categorical variables were expressed as counts and percentages.

Univariate and multivariate data analysis methods were applied to the complete data set of metabolite concentrations to investigate the general structure of the metabolomics data and how the different metabolic features are related with angioplasty intervention. A paired Student's t-test was performed to investigate the metabolite differences between before and after angioplasty serum samples.

Multivariate data analysis was carried out using SIMCA-P (V.14 Umetrics AB, Umea, Sweden). To have an overview of the study population, metabolic data of sera collected before angioplasty were analysed by principal component analysis (PCA) using unit variation scaling. Effect of balloon occlusion was studied using the percentage of changes for each metabolite as variables. In this way, each patient serves as his/her own biological control, alleviating for potential clinical confounders.

Qualities of the models were described using R2 and Q2 values. $\mathrm{R} 2$, which indicates the goodness of fit, is defined as the proportion of variance in the data explained by the model. Q2, which indicates the predictability, is defined as the proportion of variance in the data predictable by the model.

\section{RESULTS}

Baseline clinical characteristics of the 34 patients with SA (76.5\% men; $70 \pm 8$ years) enrolled are shown in table 1 .

Arterial and venous serum samples, both before and after angioplasty, were collected: out of 136 blood samples, 18 (9 venous and 9 arterial) were excluded due to corruption of the sample (ie, haemolysis, unexpected refrigeration abnormalities). Therefore, both arterial and venous serum were analysed in 16 patients; isolated arterial (9) or venous (9) sample analysis was performed for 18 subjects, giving a total of 25 observations for each type of matrix (table 2). 


\begin{tabular}{ll}
\hline $\begin{array}{l}\text { Table } 1 \text { Baseline clinical characteristics of the study } \\
\text { population }\end{array}$ & Percentage \\
\hline & 76.5 \\
\hline Sex (men) & $70 \pm 8$ \\
\hline Age (average $\pm S D)$ & 20.6 \\
\hline Chronic renal failure & 61.8 \\
\hline Hypertension & 67.6 \\
\hline Dyslipidaemia & 14.7 \\
\hline Smoking habits & 23.5 \\
\hline History of coronary artery disease & 23.5 \\
\hline Obesity & 29.4 \\
\hline Diabetes mellitus & 14.7 \\
\hline Oral hypoglycaemic agents & 11.8 \\
\hline Insulin & \\
\hline
\end{tabular}

Figure 1A shows a typical ${ }^{1} \mathrm{H}-\mathrm{NMR}$ serum spectrum with identification of the main metabolite signals. A total of 41 metabolites were identified and quantified in each ${ }^{1} \mathrm{H}-\mathrm{NMR}$ serum spectrum (table 3 ). They include a range of amino acids, saccharides, energy metabolism-related molecules (pyruvate succinate, citrate, lactate, creatine, creatinine) and others (cholines, amines and amides). They were identified on comparison with the Chenomx metabolite database. The NMR-derived profile of assigned metabolites is shown in figure 1B. Most of the NMR signals in the serum spectrum were identified: only a few signals with low intensity located in overlapped regions of the spectrum are present in the difference spectrum (figure 1C).

\section{Metabolic profile of arterial and venous sera}

Metabolite concentrations of arterial and venous sera before angioplasty were used for Multivariate Data Analysis to examine the intrinsic variation in our dataset and to identify potential outliers. A PCA was initially performed using the concentration data of arterial (figure 2A, R2X:0.418, Q2:0.07) and venous (figure 2C, R2X:0.366, Q2:-0.116) samples. The score plot of both matrices before angioplasty shows that subject 2 metabolic profile behaves as an outlier of the model (figure 2A,C). Main differences were due to high levels of choline, creatinine, citrulline, urea, carnitine and O-acetylcarnitine and low levels of valine, isoleucine and leucine (figure 2B,D). Despite these differences in the metabolic profile, changes induced by angioplasty for this subject were in

Table 2 Samples distribution of arterial (A) and venous ( $V$ ) blood sampling carried out on patients

\begin{tabular}{llllll}
\hline $\begin{array}{l}\text { Total } \\
\text { number } \\
\text { of } \\
\text { patients }\end{array}$ & $\begin{array}{l}\text { Both A } \\
\text { and V } \\
\text { samples }\end{array}$ & $\begin{array}{l}\text { Only A } \\
\text { samples }\end{array}$ & $\begin{array}{l}\text { Only V } \\
\text { samples }\end{array}$ & $\begin{array}{l}\text { Total A } \\
\text { samples }\end{array}$ & $\begin{array}{l}\text { Total V } \\
\text { samples }\end{array}$ \\
\hline 34 & 16 & 9 & 9 & 25 & 25 \\
\hline
\end{tabular}

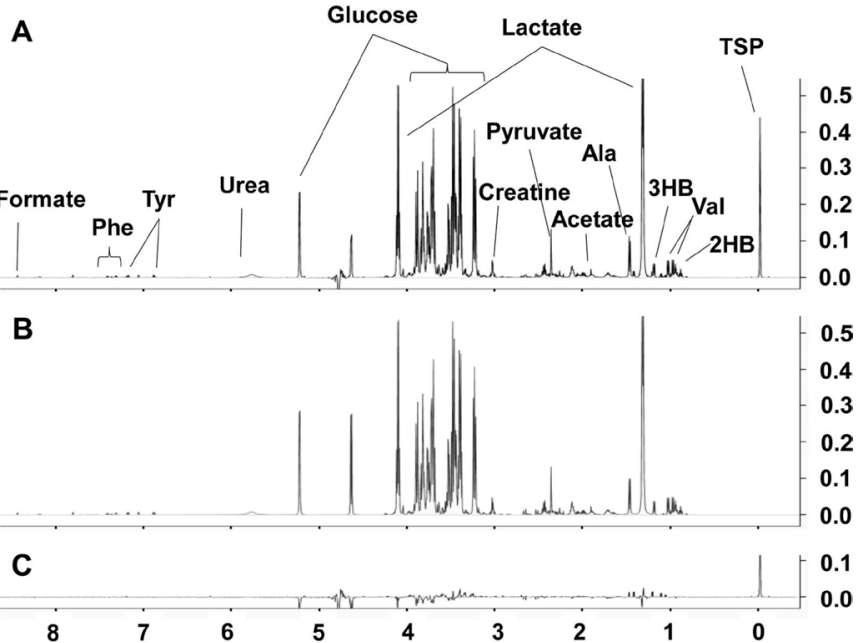

Figure 1 (A) ${ }^{1} \mathrm{H}-\mathrm{NMR}$ serum spectrum with signal assignments. (B) ${ }^{1} \mathrm{H}-\mathrm{NMR}$ spectrum of assigned metabolites. (C) difference spectrum (A, B). 2HB, 2-hydroxybutyrate; 3HB, 3-hydroxybutyrate; Ala, alanine; Gln, glutamine; Lys, lysine; Phe, phenylalanine; Tyr, tyrosine; Val, valine.

line with those observed for the entire set, so it was kept in the following analysis.

\section{Metabolic changes induced by angioplasty}

Samples collected before and after the clinical intervention on patients with SA were used to study the effect of angioplasty on arterial and venous sera. For each subject, the percentage change of each metabolite was calculated and used as input data for a PCA. In this analysis, variables are not centred, allowing for the evaluation of the variable changes. In the absence of treatment effect, subjects would appear as a cluster around the origin in the score plot. Treatment effects cause the subjects' movement from the origin.

Our results reveal that metabolic changes induced by angioplasty are clearly measurable in arterial serum, showing most of observables moving along t1 (figure $3 \mathrm{~A}$ ). This trend is also observed in the score plot of venous samples, even if the effect is much reduced (figure 3C). In both analyses, loading plots indicate that levels of ketone bodies increase significantly after angioplasty (figure 3B,D).

We finally examined significant changes $(\mathrm{P}<0.01)$ in metabolites concentration induced by balloon occlusion (table 4 and figure 4 ). In both types of serum, an increase in ketone bodies, 2-hydroxybutyrate, glutamine and $\mathrm{O}$-acetylcarnitine concentration is observed, while alanine, lactate, phenylalanine and tyrosine decreased after intervention. Most significant metabolic changes are detected in arterial serum, in line with the multivariate analysis (MVA) result.

\section{DISCUSSION}

The present study showed that a significant early systemic metabolic response to angioplasty-induced ischaemia performed in patients with SA could be identified. Specifically, an increase of ketone bodies and a simultaneous 
Table 3 Serum metabolite concentrations in arterial and venous sera collected before and after angioplasty ( $\mu \mathrm{M})$

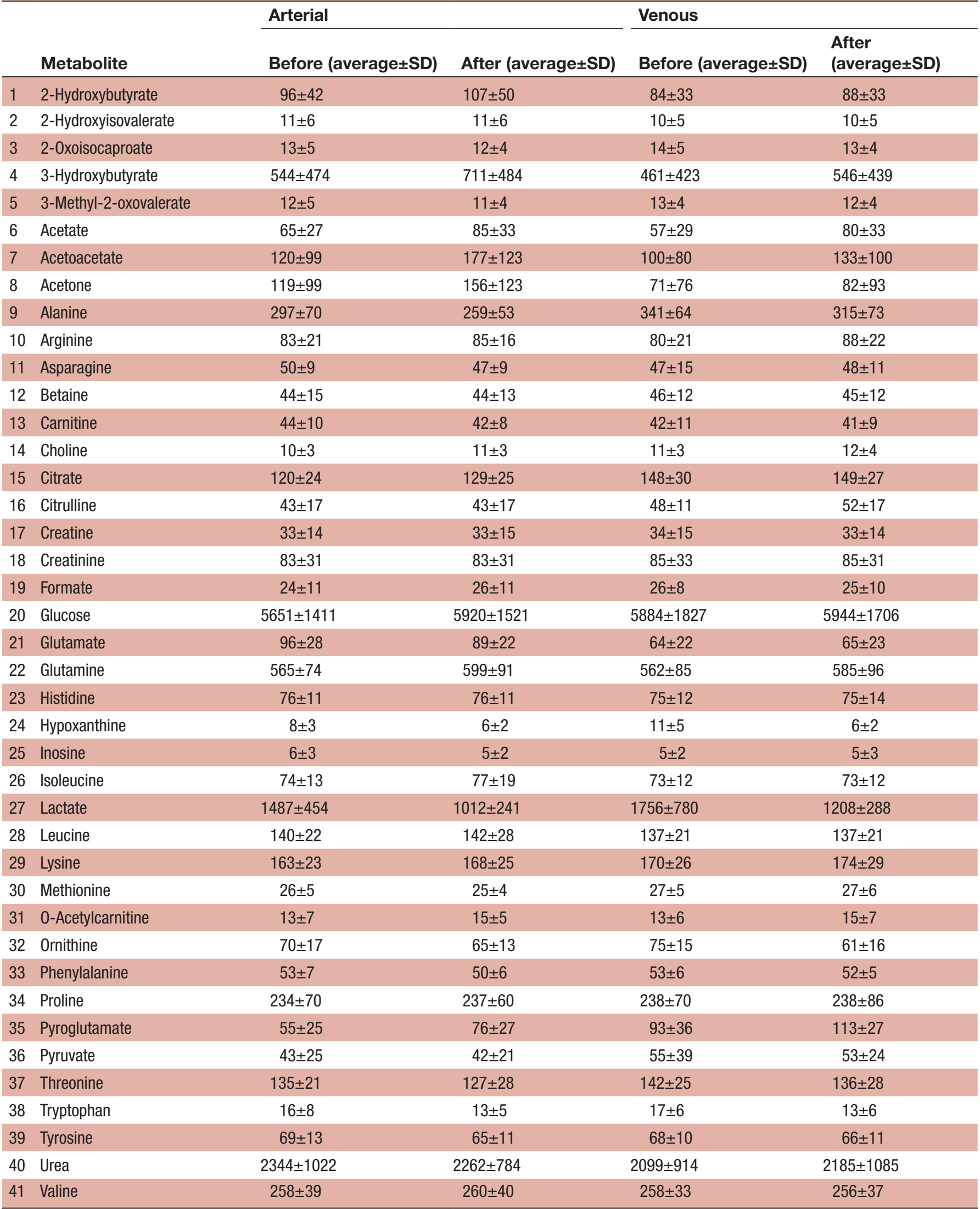



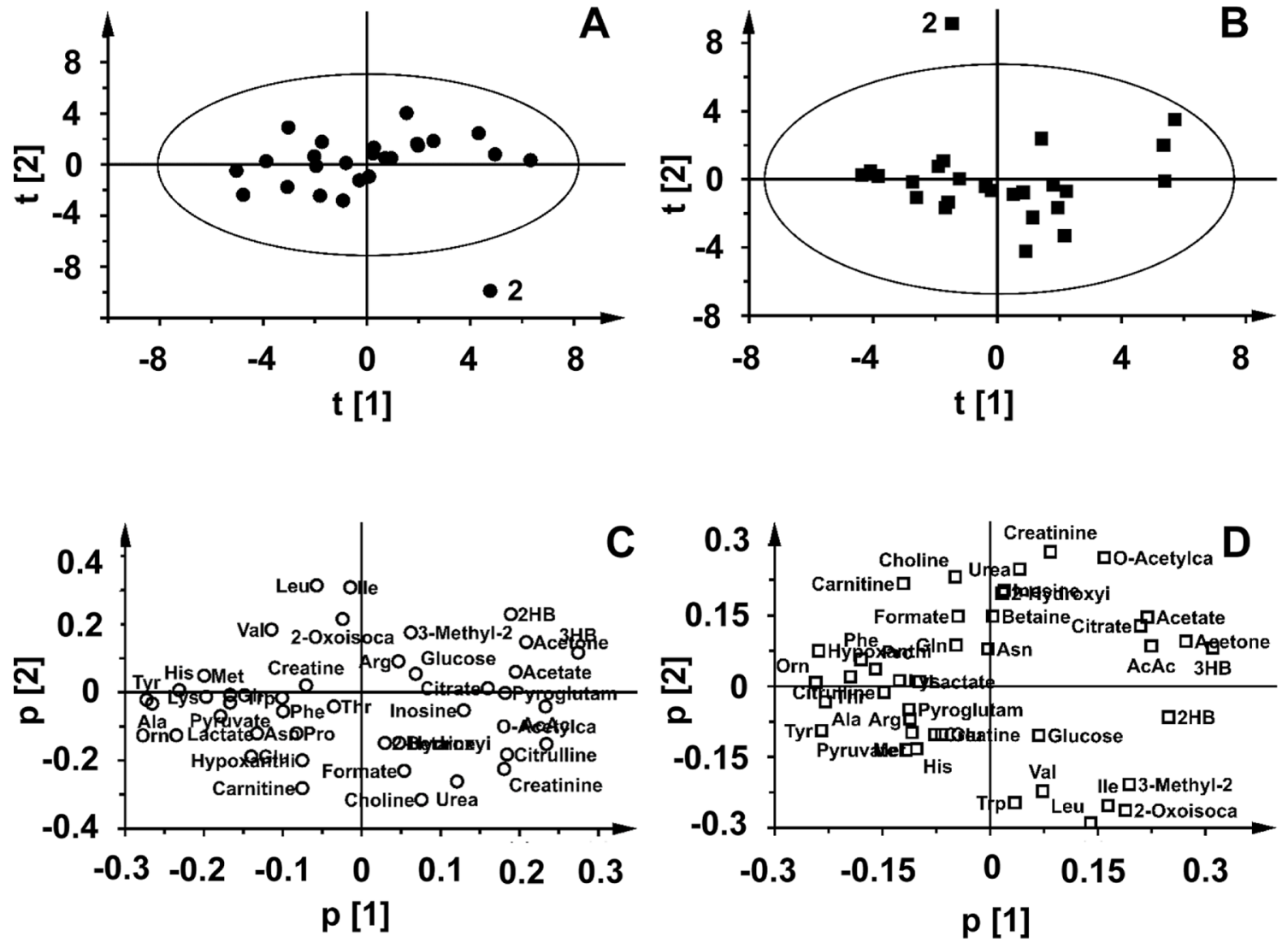

Figure 2 Principal component analysis score and loading plots of arterial $(A-C)$ and venous $(B-D)$ serum samples of samples collected before angioplasty. The ellipse shows the $95 \% \mathrm{Cl}$ (Hotelling's score) of the model. Sample corresponding to subject 2 can be considered an outlier.

decrease in lactate concentration were detected after PCI regardless of age, sex or the presence of the main cardiovascular comorbidities.
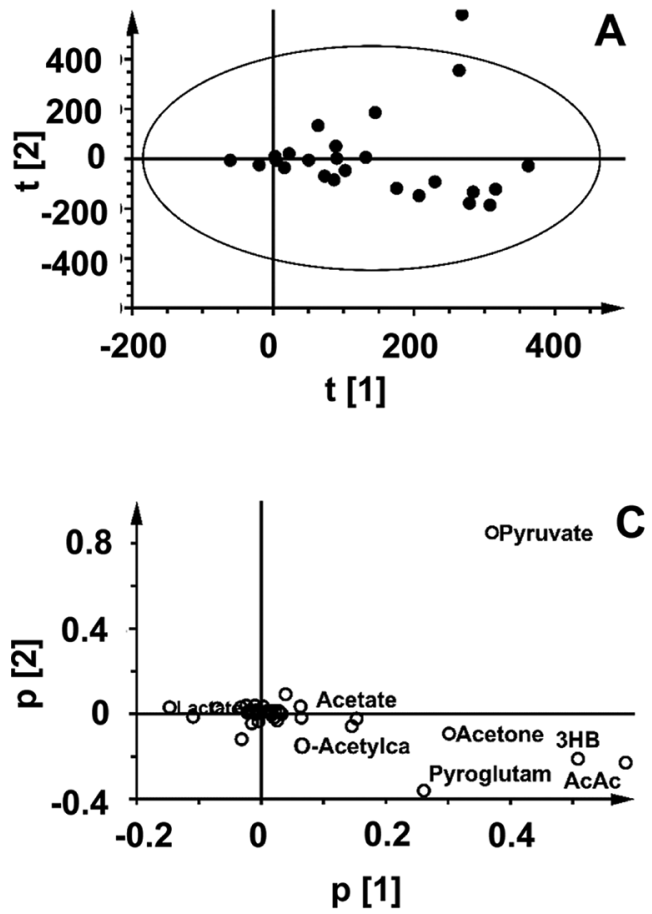

Balloon occlusion mimics ischaemia's first phase, causing a lack of oxygen availability for at least 1 min to about $20 \%-30 \%$ of the overall myocardial tissue. Our
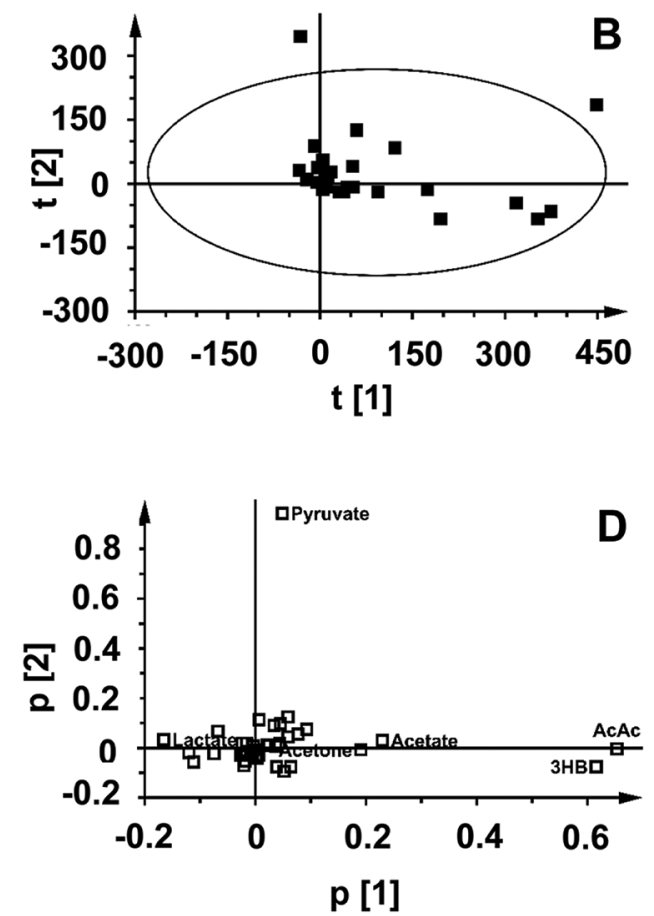

Figure 3 Change percentage induced by angioplasty: (after)-(before)/(before) $\times 100$. Principal component analysis score and loading plots of arterial $(A-C)$ and venous $(B-D)$ serum samples. 
Table 4 Metabolite changes in arterial and venous sera after angioplasty

\begin{tabular}{lllcr}
\hline \multirow{2}{*}{ Metabolite } & Arterial change & \multicolumn{2}{l}{ Venous change } \\
\cline { 2 - 3 } \cline { 5 - 5 } & & \multicolumn{2}{c}{ P values } & P values \\
\hline 2HB & $10(1$ to 16$)$ & $<0.01$ & $5(-1$ to 11$)$ & $<0.01$ \\
3HB & $56(1$ to 124$)$ & $<0.01$ & $3(-2$ to 60$)$ & 0.03 \\
Acetate & $25(9$ to 44$)$ & $<0.01$ & $23(-2$ to 78$)$ & 0.19 \\
AcAc & $55(18$ to 167$)$ & $<0.01$ & $17(-8$ to 38$)$ & 0.03 \\
Acetone & $25(16$ to 74$)$ & $<0.01$ & $-4(-9$ to 1$)$ & 0.14 \\
Ala & $-10(-19$ to -6$)$ & $<0.01$ & $4(2$ to 8$)$ & $<0.01$ \\
Gln & $6(2$ to 11$)$ & $<0.01$ & $-21(-37$ to -11$)$ & $<0.01$ \\
Lactate & $-29(-42$ to -14$)$ & $<0.01$ & $19(2$ to 31$)$ & 0.02 \\
0-Acetylcarnitine & $18(10$ to 42$)$ & 0.01 & $-4(-8$ to 3$)$ & 0.04 \\
Phe & $-6(-9$ to 0$)$ & $<0.01$ & $-4(-6$ to 0$)$ & 0.01 \\
\hline Tyr & $-5(-8$ to 1$)$ & 0.01 &
\end{tabular}

Change values denote median percentage change; values in parentheses represent IQR.

AcAc, acetoacetate; 2HB, 2-hydroxybutyrate; 3HB, 3-hydroxybutyrate; Ala, alanine; Gln, glutamine; Phe, phenylalanine; Tyr, tyrosine.

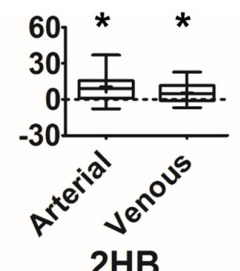

$2 \mathrm{HB}$

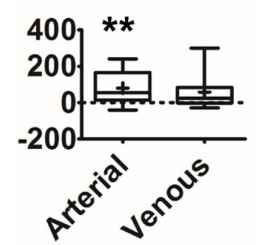

AcAc

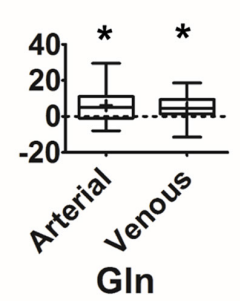

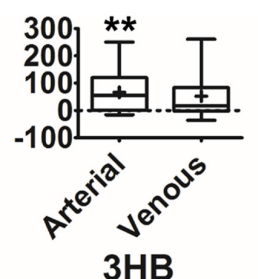
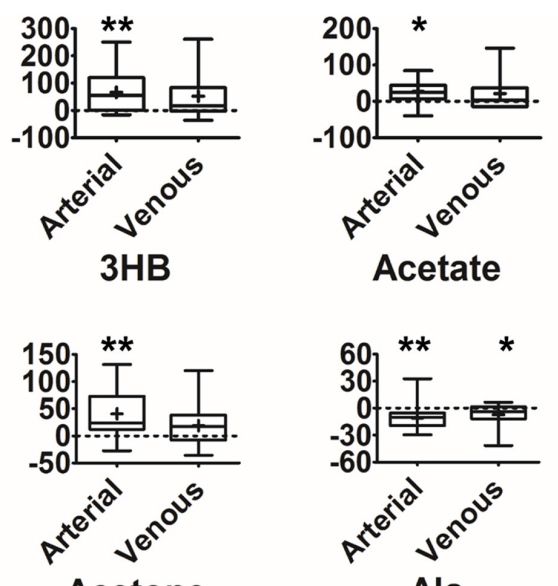

Acetone

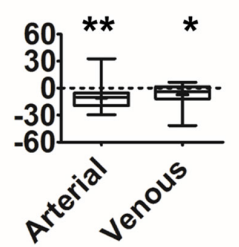

Ala
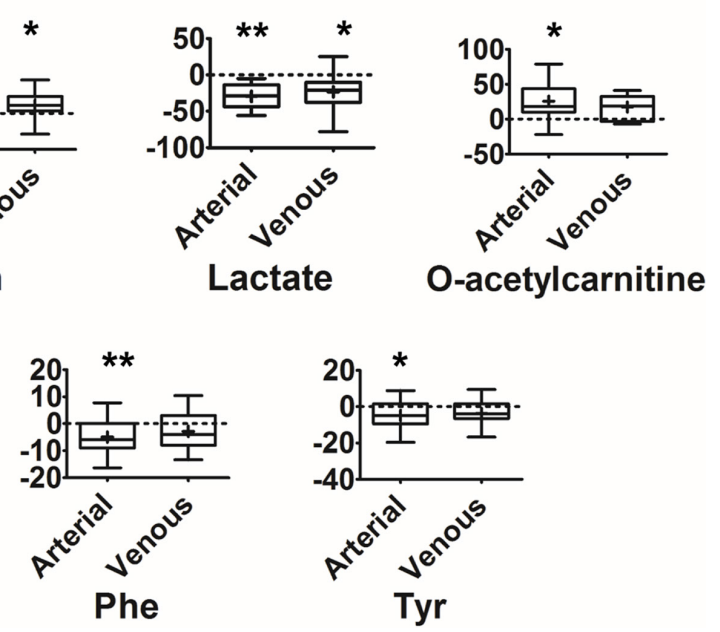

Figure 4 Change percentage induced by angioplasty in arterial $(A)$ and venous $(V)$ serum metabolites. Boxes denotes IQR, lines denotes median, whiskers denotes 5 th and 95th percentile, +denotes the average. ${ }^{*} \mathrm{P}<0.01 ;{ }^{*} \mathrm{P}<0.001 .2 \mathrm{HB}$, 2-hydroxybutyrate; 3HB, 3-hydroxybutyrate; Ala, alanine; Gln, glutamine; Lys, lysine; Phe, phenylalanine; Tyr, tyrosine; Val, valine. data show that such a local myocardial metabolic impairment is able to influence systemic biochemical changes.

\section{Lactate changes after PCI}

Lactate myocardial extraction represents an accepted gold standard for myocardial ischaemia quantification. ${ }^{6}$ Previous studies analysed changes in lactate levels after the same type of balloon-induced coronary ischaemia. ${ }^{7-9}$ In a first experiment, Gill et al measured lactate concentration in coronary sinus blood during left anterior descending coronary artery angioplasty. ${ }^{7}$ The study showed an increase of lactate concentration locally in coronary sinus blood, showing a peak that disappears 5 min after balloon deflation. Recently, Sinha et al measured lactate concentration both in coronary sinus and arterial blood, observing two different behaviours. Sinus blood lactate concentration showed a peak 1 min after balloon inflation while systemic arterial lactate concentration decreased constantly. Five minutes after balloon deflation, when coronary sinus blood lactate concentration returned to basal levels, arterial lactate was found to be $30 \%$ lower than starting concentration levels. ${ }^{8}$

In a more recent study, using NMR to analyse metabolic changes after angioplasty, surprisingly the investigators observed the opposite behaviour of this metabolite. ${ }^{9}$ Lactate in venous serum showed a 50\% increase in its concentration $10 \mathrm{~min}$ after balloon-induced ischaemia. This wide variation that is observed in circulating lactate concentration cannot be related to the proportionally small lactate production within the ischaemic myocardial tissue, but rather reflects a global response of the whole body to the local perturbation.

Our results concerning lactate variation are in contradiction with those reported by Bodi et al and in accordance with those of Shina $e t$ al. We observed a decrease in circulating lactate $10 \mathrm{~min}$ after balloon-induced ischaemia, both in arterial $(-29 \%, \mathrm{P}<0.001)$ and venous serum $(-21 \%, \mathrm{P}<0.01)$. Although we also used NMR to 
quantify metabolite levels, there is a difference in the sample preparation that could account partially for the discrepancy. We followed a well-established protocol that includes a filtration step that eliminates high-molecular-weight molecules, including lipoproteins. ${ }^{10} \mathrm{NMR}$ lactate quantification is less accurate in presence of proteins due to overlap with broad signals arising from high-molecular-weight components.

The reason why circulating lactate concentration continues to fall after balloon occlusion despite the early rise in coronary sinus blood is far from clear. Dilution effects in the systemic blood and rapid elimination/ uptake of lactate from the peripheral circulation were previously invoked. ${ }^{8}$ In our view, a positive correlation between blood lactate levels and lactate extraction by the heart could account for this behaviour. ${ }^{11}$ This fact, thoroughly studied in the context of exercise routines in healthy trained subjects, leads to a model in which arterial lactate concentration plays a central role in regulating myocardial substrate utilisation. ${ }^{12}$ During exercise, heart lactate uptake increases, leading to a fast systemic response and consequently a significant increment in circulating arterial lactate of almost $100 \%$ after 5 min. ${ }^{13}$ Interestingly, one subject in that study showed a reduction in the circulating lactate during moderate exercise. In this particular case, decreasing of arterial lactate concentration was related to lower myocardial lactate utilisation. This is the same scenario expected for the angioplasty-induced ischaemia. Lactate extraction by the heart changes from $30 \%$ before balloon inflation to a net release $(-7 \%)$ $1 \mathrm{~min}$ after occlusion and returns to a lower extraction of $16 \% 5 \mathrm{~min}$ after. $^{8}$ One could hypothesise that these variations in lactate requests by the heart are communicated to the whole body through an unknown signal, determining circulating lactate levels drop in order to meet heart's lactate extraction needs. This can constitute an adaptive mechanism similar and opposite to that one already accepted as a response to physical exercise. ${ }^{12}$

\section{Ketone bodies changes after PCI}

Significant increase in the concentration of ketone bodies (3-hydroxybutyrate and acetoacetate) that were observed in the arterial serum after angioplasty can play a role in response to the metabolic imbalance produced by ischaemia or to the subsequent reperfusion. Higher concentrations of circulating ketone bodies may counterbalance the augmented glycolysis caused by balloon-induced ischaemia. The heart is the highest ketone body consumer per unit mass, oxidising ketone bodies in proportion to their delivery, decreasing fatty acid and glucose oxidation. ${ }^{14-16}$ Elevated plasma ketone bodies levels inhibit glucose and lactate, uptake and oxidation presumably through pyruvate dehydrogenase (PDH) inhibition. ${ }^{17}$ Related to this pathway, we observed also circulating O-acetylcarnitine and acetate increasing after angioplasty. This finding could reflect acetyl-CoA production imbalance, the final product of $\mathrm{PDH}$ from pyruvate, which the carnitine-acetylcarnitine couple contributes to buffer. ${ }^{18}$ Acetate is quickly metabolised into acetyl-CoA, which is then used by the myocardiocytes in the TCA cycle. $^{19}$

On the other hand, increased levels of ketone bodies could be a response to reperfusion oxidative stress. Although the regulation mechanism of myocardial ROS is not completely understood, it is thought that high levels of ketone bodies maintain ubiquinone in the oxidised state, increasing redox span in the electron transport chain. ${ }^{20}$ This, in turn, diminishes superoxide production and increases energy availability for ATP synthesis. ${ }^{21}$ All these data suggest that ketone bodies oxidation may play an important role in free radical homeostasis during ischaemia-reperfusion injury. ${ }^{20}$

\section{Clinical implication and limitations}

The study reported has pathophysiological and clinical implications. The systemic early metabolic response observed in patients with SA after PCI might represent an overall compensation of the metabolic system to counteract the lack of metabolic substrates in the ischaemic myocardial tissue. This phenomenon leads onto the hypothesis of systemic cross-talk among peripheral tissues in response to stress events (ie, ischaemia) aimed at maintaining functioning tissues. Identification of a specific metabolic change induced by myocardial ischaemia could be of clinical relevance to rule out coronary events in patients referring for acute chest pain in emergency departments. However, risk stratification in patients with chest pain needs a statistic model to be developed by collecting wide sample of population.

Basal metabolic profile of each subject is influenced by factors like sex, age, diet, drugs and comorbidities. Collecting, in the same patient, samples both before and after a controlled ischaemia allows to override this heterogeneity, thus providing to each patient his own control.

Metabolic response following myocardial ischaemia changes very quickly, providing an instant picture of the whole-body response. Thus, serial peripheral blood sampling could help to better understand metabolic changes over time.

Finally, since lactate production is mainly muscular workout related, it is crucial to further validate this metabolite as a marker in the setting of exercise stress-induced ischaemia.

\section{Conclusions}

Early metabolic response to myocardial ischaemia has not been completely investigated despite improvements in metabolomics technologies. Our proof-of-concept study shows that a systemic biochemical profile defined by two main metabolic changes in peripheral blood can describe events following balloon-induced ischaemia: ketone bodies increase and lactate decreases. Such systemic metabolic variations may be related to compensation mechanisms aiming to fulfil the heart's needs after short period of myocardial ischaemia, and probably after reperfusion. We believe that these findings may potentially help 
identify and target new markers of very early myocardial acute ischaemia and may prove a stepping stone towards improving the accuracy of current gold standard diagnostic tools for ischaemic heart disease.

Contributors GT, NV and DOC made substantial contributions to the conception and design of the work. SDM, AL, GT and DOC made substantial contributions to the acquisition, analysis or interpretation of data for the work. SDM, AL, VS, GT, NV, VR, EM, CG and DOC made substantial contribution to the work or revising it critically for important intellectual content and final approval of the version to be published.

Funding Collezione Nazionale di Composti Chimici e Centro Screening (CNCCS) Consortium, Grant: Project B, Sp 2, WP4, 2016 'Metabolic and risk profile of cardiovascular diseases'. Scientific Independence of Young Researchers Program (RBSI14HNVT), funded by Italian Ministry of Education, University and Research (MIUR).

Competing interests None declared.

Patient consent Obtained.

Ethics approval Code 3786 Policlinico Umberto I Roma.

Provenance and peer review Not commissioned; externally peer reviewed.

Data sharing statement № additional unpublished data are available for this study.

Open Access This is an Open Access article distributed in accordance with the Creative Commons Attribution Non Commercial (CC BY-NC 4.0) license, which permits others to distribute, remix, adapt, build upon this work non-commercially, and license their derivative works on different terms, provided the original work is properly cited and the use is non-commercial. See: http://creativecommons.org/ licenses/by-nc/4.0/

C Article author(s) (or their employer(s) unless otherwise stated in the text of the article) 2018. All rights reserved. No commercial use is permitted unless otherwise expressly granted.

\section{REFERENCES}

1. World Health Organization. In: Mendis S, Puska P, Norrving B, eds Global Atlas on Cardiovascular Disease Prevention and Control. Geneva: World Health Organization, 2011.

2. Kavsak PA, Wang X, Henderson M, et al. PAPP-A as a marker of increased long-term risk in patients with chest pain. Clin Biochem 2009;42:1012-8.

3. Bodí V, Sanchis J, Llàcer A, et al. Multimarker risk strategy for predicting 1-month and 1-year major events in non-ST-elevation acute coronary syndromes. Am Heart J 2005;149:268-74.

4. Dieterle F, Ross A, Schlotterbeck G, et al. Probabilistic quotient normalization as robust method to account for dilution of complex biological mixtures. Application in $1 \mathrm{H}$ NMR metabonomics. Anal Chem 2006;78:4281-90.

5. Sim J, Lewis M. The size of a pilot study for a clinical trial should be calculated in relation to considerations of precision and efficiency. $J$ Clin Epidemiol 2012;65:301-8.

6. Serruys PW, Wijns W, van den Brand M, et al. Left ventricular performance, regional blood flow, wall motion, and lactate metabolism during transluminal angioplasty. Circulation 1984;70:25-36.

7. Gill JS, Prasad K, Stewart JT, et al. Acute changes in atrial natriuretic peptide, insulin-like growth factor-1, and lactate levels during left anterior descending coronary artery angioplasty. Am Heart $J$ 1995;130:717-22.

8. Sinha MK, Vazquez JM, Calvino R, et al. Effects of balloon occlusion during percutaneous coronary intervention on circulating Ischemia Modified Albumin and transmyocardial lactate extraction. Heart 2006;92:1852-3.

9. Bodi V, Sanchis J, Morales JM, et al. Metabolomic Profile of Human Myocardial Ischemia by Nuclear Magnetic Resonance Spectroscopy of Peripheral Blood Serum. J Am Coll Cardiol 2012;59:1629-41.

10. Psychogios N, Hau DD, Peng J, et al. The human serum metabolome. PLoS One 2011;6:e16957.

11. Gertz EW, Wisneski JA, Neese R, et al. Myocardial lactate extraction: multi-determined metabolic function. Circulation 1980;61:256-61.

12. Adeva-Andany $M$, López-Ojén $M$, Funcasta-Calderón $R$, et al. Comprehensive review on lactate metabolism in human health. Mitochondrion 2014;17:76-100.

13. Gertz EW, Wisneski JA, Stanley WC, et al. Myocardial substrate utilization during exercise in humans. Dual carbon-labeled carbohydrate isotope experiments. J Clin Invest 1988;82:2017-25.

14. Tardif A, Julien N, Pelletier A, et al. Chronic exposure to betahydroxybutyrate impairs insulin action in primary cultures of adult cardiomyocytes. Am J Physiol Endocrinol Metab 2001;281:E120 5-E1212.

15. Hasselbaink DM, Glatz JF, Luiken JJ, et al. Ketone bodies disturb fatty acid handling in isolated cardiomyocytes derived from control and diabetic rats. Biochem J 2003;371:753-60.

16. Crawford PA, Crowley JR, Sambandam N, et al. Regulation of myocardial ketone body metabolism by the gut microbiota during nutrient deprivation. Proc Natl Acad Sci U S A 2009;106:11276-81.

17. Stanley WC, Recchia FA, Lopaschuk GD. Myocardial substrate metabolism in the normal and failing heart. Physiol Rev 2005;85:1093-129.

18. Stephens FB, Constantin-Teodosiu D, Greenhaff PL. New insights concerning the role of carnitine in the regulation of fuel metabolism in skeletal muscle. J Physiol 2007;581:431-44.

19. Grassi I, Nanni C, Allegri V, et al. The clinical use of PET with (11) C-acetate. Am J Nucl Med Mol Imaging 2012;2:33-47.

20. Schugar RC, Moll AR, André d'Avignon D, et al. Cardiomyocytespecific deficiency of ketone body metabolism promotes accelerated pathological remodeling. Mol Metab 2014;3:754-69.

21. Veech RL. The therapeutic implications of ketone bodies: the effects of ketone bodies in pathological conditions: ketosis, ketogenic diet, redox states, insulin resistance, and mitochondrial metabolism. Prostaglandins Leukot Essent Fatty Acids 2004;70:309-19. 